\title{
UTERINE SARCOMAS - CURRENT THERAPEUTIC OPTIONS
}

\author{
N. Bacalbașa ${ }^{1}$, A. Traistaru², I. Bălescu ${ }^{3}$ \\ 1“Carol Davila " University of Medicine and Pharmacy, Bucharest, Romania \\ ${ }^{2}$ Elias Emergency University Hospital, Bucharest, Romania \\ 3"Ponderas" Hospital, Bucharest, Romania
}

\begin{abstract}
Uterine sarcomas are rare but very aggressive uterine malignancies originating from mesenchymal elements of the uterine wall. They are classified by the "Gynecologic Oncology Group" in nonepithelial neoplasms and mixed epithelial-nonepithelial tumors, each category presenting different features and prognostic factors. In this review we present the main characteristics and the current therapeutic options for each type of primary uterine sarcoma and for their recurrences.
\end{abstract}

Key words: uterine sarcoma, surgery, recurrence

\section{Introduction}

Uterine sarcomas are rare tumors accounting for $3-7 \%$ of all uterine neoplasms (1), with poor prognosis. Uterine sarcomas originate from mesenchymal elements at the level of the uterine wall: endometrial stroma and myometrium smooth muscle tissue.

\section{Classification of uterine sarcomas}

Uterine sarcomas were histologically classified by the "Gynecologic Oncology Group" as it follows: $(1,2)$

I. Nonepithelial neoplasms

A.Endometrial stromal tumors

1. Stromal nodule

2. Low-grade stromal sarcoma
3. High-grade stromal sarcoma

B. Smooth muscle tumor of uncertain malignant potential

C. Leiomyosarcoma

1. Epithelioid

2. Myxoid

D.Mixed endometrial stromal and smooth muscle tumor

E. Poorly differentiated (undifferentiated) endometrial sarcoma

F. Other soft tissue tumors

1. Homologous

2. Heterologous

II. Mixed epithelial-nonepithelial tumors
A. Adenosarcoma
1. Homologous
2. Heterologous

Address for correspondence: Nicolae Bacalbașa, MD

Dimitrie Racoviță Street, no. 2, Bucharest, Romania, e-mail: nicolae_bacalbasa@yahoo.ro 
3. With high-grade stromal overgrowth (see notes)

B. Carcinosarcoma (malignant mixed mesodermal tumor or malignant mixed müllerian tumor)

\section{Homologous}

2. Heterologous

Homologous histological types contain elements that are specific to uterine tissue (Spindle cell sarcoma, stromal sarcoma, leiomyosarcoma, Fibrosarcoma) while heterologous ones contain histological elements that usually do not exist at this level (osteosarcoma, chondrosarcoma, rhabdomyosarcoma). (1)

The incidence of uterine sarcomas in the United States is $2.68 / 100000$. The most common types of uterine sarcomas are in order of frequency carcinosarcoma, leiomyosarcoma, endometrial stromal sarcoma and adenosarcoma. Both type and incidence of uterine sarcomas are dependent on age and race. Carcinosarcoma rarely occurs before age 40 , the mean age of patients with this type of tumor being 65 while leiomyosarcoma occurs in younger patients, the mean age being 55 years. (1)

\section{FIGO staging for uterine sarcomas (2009)}

Stage I (tumor limited to uterus)

IA: $<5 \mathrm{~cm}$ tumor size

IB: $\geq 5 \mathrm{~cm}$ tumor size

For Leiomyosarcoma

IA: tumor limited to endometrium/ endocervix without myometrial invasion

IB: tumor invades $<50 \%$ myometrium

IC: tumor invades $>50 \%$ myometrium

For Endometrial Stromal Sarcoma and Adenosarcoma

Stage II (tumor extension into the pelvis)

IIA: adnexal involvement

IIB: other extrauterine pelvic disease

Stage III (tumor invades abdominal tissues)

IIIA: one site involvement

IIIB: $>1$ site involvement

IIIC: metastasis to pelvic and/or para-aortic lymph nodes

Stage IV (tumor invades bladder and/or rectum, and/or distant metastasis)

IVA: involvement of bladder and/or rectum

IVB: distant metastasis

\section{Uterine carcinosarcomas}

Carcinosarcoma contains both malignant epithelial and mesenchymal elements. Epithelial component is usually represented by serous and endometrioid cells, and rarely, mucinous or squamous cells while mesenchymal component is heterogeneous. The most important prognostic factor in carcinosarcoma is tumor stage; the 5 year overall survival is 59\%, $22 \%$ and $9 \%$ for stages I, II and III respectively. Other prognostic factors are the cell type, presence or absence of lymphatic spread, grade of differentiation and number of mitoses, sarcomatous component, depth of myometrial invasion, peritoneal cytology. $(3,4)$

Surgical treatment for patients with uterine carcinosarcoma is total hysterectomy with bilateral adnexectomy with pelvic and para-aortic lymph node dissection. Staging is performed according to the FIGO staging system for endometrial cancer. Carcinosarcomas re tumors with a high capacity of extension: $37 \%$ of patients present myometrail invasion, $17 \%$ present nodal involvement and almost $21 \%$ of patients present malignant cells in the peritoneal lavage cytology. $(3,4,5)$

In almost all cases the presence of the distant metastases is due to the epithelial component of the carcinosarcoma.

In a study conducted on 3962 cases diagnosed histopatologicaly with carcinosarcoma, lymph node dissection was performed in 53\% of cases. 5-year survival rate in cases without extrapelvic extension, was $59 \%$ for IA stage, $54 \%$ for IB stage and only $38 \%$ for IC stage. In another study of the Gynecologic Oncology Group, 53\% of all patients with carcinosarcoma and $40 \%$ of those with stage I carcinosarcoma had a recurrence in the first 3 years after diagnosis. The prognosis depends on the presence of lymph node metastasis, depth of myometrial invasion and impaired uterine segment or cervix. (6)

Adjuvant therapy is represented by adjuvant radiotherapy or chemotherapy in order to obtain a good local control of the disease. Radiation therapy is usually used to reduce the frequency of pelvic recurrences. Several studies have shown a decrease in local recurrence, but no evidence of any benefit in terms of survival. Recently, a study made by the European Organization for Research and Treatment of Cancer (EORTC) on 224 patients diagnosed with uterine sarcoma (91 cases of carcinosarcoma) showed that pelvic radiotherapy after surgery does not improve survival, but in cases of carcinosarcoma, decreases the risk of pelvic recurrence. (1) 


\section{Uterine leiomyosarcomas}

Leiomyosarcoma is a malignant tumor originating from the smooth muscle cells of the myometrium. In these cases preoperative diagnosis is usually difficult to be established; they are often cathegorised as benign leiomyomas; the only preoperative examination which could differentiate the two histopathological types is the tumor tissue biopsy. Macroscopically, quite often the tumor borders are irregular, infiltrating the surrounding tissue. The main histopathological features necessary to diagnose leiomyosarcoma are: cellular atypia, a large number of mitoses and the presence of areas of coagulation necrosis. (1)

The most common way of dissemination is the hematogenous route, approximately $10 \%$ of patients presenting lung metastases at the moment of presentation. $(5,6)$

The correlation between mitotic index, tumor type and metastatic potential is shown in the table 1. (7)

The standard treatment of uterine leiomyosarcomas in premenopausal women is total interadnexial hysterectomy; unfortunately there are cases in which the right diagnosis is not known preoperatively and a myomectomy is performed for the preoperative suspicion of benign tumor. In these cases most authors opiniate for a second surgery in order to perform a complete hysterectomy.

Prognostic factors - the most important prognostic factor in leiomyosarcoma is the stage of the disease. The 5 year survival rate is about 50\% for stage I-II. (table 2)

Lymph node metastases have a low incidence in leiomyosarcomas (2.7 to $4 \%$ ). In the study of Giuntoli et al on 208 patients with leiomyosarcoma, lymph node metastasis were found in 4 of 36 patients who underwent lymph node dissection, while three of these patients presented multiple metastases. There seems to be a significant difference in survival in patients with stage I leiomyosarcoma with or without lymph node metastases. $(8,9)$

Older age at diagnosis is a poor prognostic factor. In a retrospective study conducted in Italy, patients aged over 50 years old had a risk of progression of the disease 2.07 times higher than patients younger than 50 years while $\mathrm{Wu}$ et al showed that patients over 50 years old had a risk of death of 7.11 times when compared to younger patients. (10)

Tumor size is another important prognostic factor, especially in stage I disease. Abeler et al reported a 5-year survival rate of $64 \%$ in cases presenting a tumor smaller than $50 \mathrm{~cm}, 56 \%$ in cases with tumors measuring between 50 and $100 \mathrm{~mm}$-, and only 29\% in cases with tumors larger than $100 \mathrm{~mm}$. Combining size tumor with the mitotic index, led to the classification of patients into three risk groups: low risk group (tumor size less than $100 \mathrm{~mm}$ and less than 10 mitoses / 10 high-resolution fields), medium-risk group (whether the tumor is larger than $100 \mathrm{~mm}$, and no more than 10 mitoses / 10 fields) - risk of death 1.9 times higher, and high-risk group (tumor greater than $100 \mathrm{~mm}$ or a high index of mitosis) - risk of death by 5.3 times higher. $(3,11,12)$

\section{Endometrial stromal sarcomas}

Endometrial stromal tumors are tumors arising from mesenchymal cells from the uterine mucosa. The macroscopic aspect of the tumors can be soft, fleshy, smooth and sometimes polypoid. These tumors are classified in two groups according to

Table 1

\begin{tabular}{lccc}
\hline Mitotic figures/10 hfh & Atypia & Tumor type & Metastatic potential \\
\hline $1-4$ & Any degree & Leiomyoma & Very low \\
\hline $5-9$ & None & Leiomyoma with high mitotic activity & Very low \\
\hline $5-9$ & Grade 1 & Smooth muscle tumor & Low \\
& & of uncertain malignant potential & \\
& & („STUMP”) & Moderate \\
$5-9$ & Grade 2 or 3 & Leiomyosarcom & High \\
$\geq 10$ & Grade 1 & Leiomyosarcom & Very high \\
\hline 10 & Grade 2 or 3 & Leiomyosarcom &
\end{tabular}


Table 2

\begin{tabular}{lccc}
\hline Mitotic figures/10 hfh & Atypia & Tumor type & Metastatic potential \\
\hline $1-4$ & Any degree & Leiomyoma & Very low \\
\hline Clinical trial & Stage & Number of pacients & Survival rates after 5 years \\
\hline Kapp (3) & I & 951 & $75,8 \%$ \\
& II & 43 & $60,1 \%$ \\
& III & 99 & $44,9 \%$ \\
Mayerhofer (11) & IV & 303 & $28,7 \%$ \\
& I & 49 & $75 \%$ \\
Salazar Dunne (4) & II & 5 & $14 \%$ \\
& III-IV & 17 & $0 \%$ \\
Gadducci (2) & I & 113 & $53 \%$ \\
& II-IV & 50 & $8 \%$ \\
\hline Kahanpaa (5) & I-II & 90 & $54 \%$ \\
& III & 16 & $6 \%$ \\
Blom (13) & IV & 20 & $0 \%$ \\
& I & 23 & $74 \%$ \\
\hline
\end{tabular}

their potential for metastasis: stromal nodules benign tumors that can not be differentiated from the endometrial stroma and stromal sarcomas tumors presenting a local invasiveness with vascular and lymphatic extension. $(2,14)$

Endometrial stromal sarcomas are classified on the basis of mitotic activity in sarcomas with low mitotic index ("low-grade"), and sarcomas with increased mitotic index or high-grade sarcoams. Tumors with low mitotic activity have an infiltrative pattern of development, their macroscopic appearance is a sinuous route being developed in the myometrium or in the pelvic blood vessels. Microscopically, these tumors present cellular atypia, with low mitotic index; although metastasis might appear, endometrial stromal sarcomas have a rather favorable clinical outcome. Recurrence usually appears after a long disease free period, of over 5 years although relapse can occur even at 25 years after the initial diagnosis. Survival at 80 months after diagnosis is about 90\%. (1)

Endometrial stromal sarcomas with high rate of cell mitosis ("high grade") have a more aggressive evolution. In a study conducted on a sample of 109 cases, Kempson et al demonstrated that the tumor stage is a more precise prognostic factor than the rate of mitosis. (15)

In another study, on 114 patients with endometrial stromal sarcoma , enroled from 1990 to 2012 , showed that in the premenopausal patients bilateral salpingo-oophorectomy was independently associated with longer recurence-free survival. Patients who underwent cytoreductive resection of recurrent tumor demonstrated longer survival after recurrence than patients without this surgery. The incidence of nodal metastasis among patients with endometrial stromal sarcomas with lymphadenectomy was 6,6\%. Endometrial stromal sarcomas show a higher incidence of estrogen and progesterone receptors compared with other uterine sarcomas. Based on the hormonal sensitiviy of endometrial stromal sarcomas and risk for recurrence, treatment of endometrial stromal sarcoma consist of performing bilateral salpingo-oophorectomy. Bilateral salpingo-oophorectomy should be considered a primary surgical treatment for improving the recurrence free survival. (2)

Cytoreductive procedures of recurrent endometrial stromal sarcoma is an independent predctor of survival after recurrence. Of the 28 patients who underwent cytoreductive surgery, 6 patients died and 22 were succesfully salvaged. Aggressive and repeated surgical resection of recurrent tumors seemed to be valuable. The 5 years overall survival rate for all the patients was $92,6 \%$. In FIGO stage I was $96,1 \%$ and in FIGO II-IV $76 \%$. The recurrence was $28,9 \%$ and the death rate was $8,7 \%$. $(7,8,15,16,17)$

\section{Conclusion}

Uterine sarcomas are relatively uncommon 
tumors that usually have an aggressive clinical behaviour and a poor prognosis. The only curative treatment remains surgical - total abdominal hysterectomy and bilateral salpingo-oophorectomy. Pelvic and para-aortic lymphadenectomy is indicated for carcinosarcoma but not for leiomyosarcoma and undifferentiated endometrial sarcoma. Adjuvant pelvic radiotherapy appears to improve local controlwithout any significant impact on overall survival. Uterine sarcomas have a high tendency to develop distant recurrences. The treatment of recurrent disease often requires the integration of different therapeutic modalities. No curative therapeutic option is currently available except surgery for isolated pulmonary metastases or association between hormone therapy and debulking surgery. $(2,14)$

\section{References}

1. McMeekin. S., Sarcoma of the uterus. Clinical Gynecologic Oncology, Eighth Edition (2012) 175-187.e3. doi:10.1016/ B978-0-323-07419-3.00006-0

2. Angiolo Gadducci. Prognostic factors in uterine sarcoma. Best Practice and Research Clinical Obstetrics and Gynecology 25 (2011) 783-795.

3. Kapp DS, Shin JY, Chan JK. Prognostic factors and survival in 1396 patients with uterine leiomyosarcomas: emphasis on impact of lymphadenectomy and oophorectomy. Cancer 2008;112:820-30.

4. Salazar OM \& Dunne ME. The role of radiation therapy in the management of uterine sarcomas. Int $\mathrm{J}$ Radiat Oncol Biol Phys 1980; 6: 899-902.
5. Kahanpaa KV, Wahlstrom T, Grohn P et al. Sarcomas of the uterus: a clinicopathologic study of 119 patients. Obstet Gynecol 1986; 67: 417-424

6. Major FJ, Blessing JA, Silverberg SG et al. Prognostic factors in early-stage uterine sarcoma. A Gynecologic Oncology Group study. Cancer 1993; 71(4 Suppl.): 1702-1709.

7. Ip PP, Cheung AN, Clement PB. Uterine Smooth Muscle Tumors of Uncertain Malignant Potential (STUMP): a clinicopathologic analysis of 16 cases. Am J Surg Pathol 2009;33:992-1005

8. Dionigi A, Oliva E, Clement PB, Young RH. Endometrial stromal nodules and endometrial stromal tumors with limited infiltration: a clinicopathologic study of 50 cases. Am J Surg Pathol 2002;26:567-81.

9. Tavassoli FA, Norris HJ. Mesenchymal tumours of the uterus. VII. A clinicopathological study of 60 endometrial stromal nodules. Histopathology 1981;5:1-10.

10. Li, N., Wu, L., Zhang, H., An, J., Li, X. and Ma, S.( 2008) Treatment options in stage I: Leiomyosarcoma: a retrospective analysis of 53 cases. Gynecol Oncol 108: 306-311.

11. Mayerhofer K, Obermair A, Windbichler G, et al. Leiomyo-sarcoma of the uterus: a clinicopathologic multicenter study of 71 cases. Gynecol Oncol 1999;74:196-201

12. Larson B, Silfversward C, Nilsson B, Petterson F. Prognostic factors in uterine leiomyosarcoma: a clinicopathologic study of 143 cases. The Radiumhemmet series, 1936-1981. Acta Oncol 1990;29:185-91.

13. Blom R, Guerrieri C, Stâl $O$ et al. Leiomyosarcoma of the uterus: a clinicopathologic, DNA flow cytometric, p53, and mdm-2 analysis of 49 cases. Gynecol Oncol 1998; 68: 54-61.

14. Angiolo Gadducci, Stefania Cosio, Antonella Romanini. The Management of patients with uterine sarcoma: A debated clin- ical challenge. Critical Reviews in Oncology/Hematology 65 (2008) 129-142.

15. Kempson RL, Bari W: Uterine sarcomas: Classification, diagnosis and prognosis. Hum Pathol 1: 331, 1970

16. Baker P, Oliva E. Endometrial stromal tumours of the uterus: a practical approach using conventional morphology and ancillary techniques. J Clin Pathol 2007;60:235-43.

17. Schilder JM, Hurd WW, Roth LM, Sutton GP. Hormonal treatment of an endometrial stromal nodule followed by local excision. Obstet Gynecol 1999;93:805-7 